(25) W. T. Carnall, P. R. Fields, and K. Rajnak, J. Chem. Phys., 49, 4447, $4450(1968)$

(26) C. J. Hawkins, "Absolute Configuration of Metal Complexes", Wiley-ln- terscience, New York, N.Y., 1971, Chapter 5

(27) H. F. Halliwell and S. C. Nybrug, Trans. Faraday Soc., 59, 1126 (1963).

(28) E. Nakazawa and S. Shionoya, J. Chem. Phys., 47, 3211 (1967).

\title{
"Semiempirical" Models for Biomembrane Phase Transitions and Phase Separations
}

\author{
J. A. McCammon* and J. M. Deutch' \\ Contribution from the Department of Chemistry, Harvard University, \\ Cambridge, Massachusetts 02138. Received March 31, 1975
}

\begin{abstract}
Experimental studies are reviewed to construct a model for the hydrocarbon chain disordering in "fluid" phospholipid bilayers. Together with approximate expressions for the interactions between neighboring molecules, this information is used to construct a simple statistical thermodynamic theory of the chain "melting" based on the Bragg-Williams approximation. Two unknown parameters in the theory, the interaction between neighboring chains in the "fluid" state and the change in head group interaction associated with the chain "melting", are determined for particular classes of phospholipids by reference to experimental data. The theory is applied to predict the "melting" temperatures of single-component bilayers and the phase diagrams of two-component bilayers. Possible extensions of this type of theory to rationalize other phenomena observed in biological membranes are discussed.
\end{abstract}

\section{Introduction}

The thermally induced phase transitions in phospholipid bilayers are known to involve a change in the state of the hydrocarbon chains in the interior of the bilayer. As the temperature is raised, the bilayer is transformed from a highly ordered "solid" state with the chains in all-trans conformations to a more disordered "fluid" state with some gauche rotations in the chain bonds. ${ }^{48}$

In dilute, aqueous, lamellar dispersions of highly purified phospholipids, the phase transitions occur at well-defined temperatures which depend on the kind of headgroup and the length and degree of saturation of the hydrocarbon chains. ${ }^{2,3}$ In lamellar dispersions of lipid mixtures, the transition occurs over a wider temperature range and is accompanied by the separation of fluid and solid phases of different composition within the bilayer. ${ }^{4-6}$ Such transitions have also been observed in cell membranes, where they have a profound effect on cell growth rates. ${ }^{7}$ Evidence is accumulating which implicates these transitions in a wide variety of biological control systems, including hormone receptor-enzyme interactions, ${ }^{8}$ signal transmission in nerves, ${ }^{9}$ enzyme regulation and active transport, ${ }^{10}$ and biological clocks. ${ }^{11}$ The medical importance of the physical states of membranes and other lipid assemblies has been discussed in several recent articles. ${ }^{12-16}$

Information about the details of the molecular disordering associated with bilayer phase transitions has been provided by recent thermal, spectroscopic, and diffraction studies. In this paper, we use the results of these studies to guide the development of a class of statistical thermodynamic models which describe some aspects of bilayer phase transitions and phase separations. The essential element in these models is the temperature-dependent balance between the energetically preferred solid state, in which the tightly packed all-trans hydrocarbon chains enjoy large van der Waals stabilization, and the high entropy of the fluid state, in which the more loosely packed chains enjoy greater conformational freedom.

In explicitly building experimentally derived structural ideas into our models, we depart from the path of previous theoretical work. ${ }^{17-21}$ We sacrifice the elegance of $a b$ initio construction in favor of an approach which should be accessible to a wide audience because of its simplicity and which may be easily modified to encompass a broader range of phenomena. Such statistical thermodynamic models may make possible the practical analysis of data within a simple conceptual scheme, much as helix-coil models have done in the field of protein chemistry.

Although our work is based on well-established bilayer properties where possible, we have had to resort occasionally to rather drastic assumptions where the experimental picture remains cloudy or where cumbersome theoretical refinements seemed premature. These arbitrary elements in this work, which will be discussed later in the paper, should for the most part yield to straightforward corrections with further progress in experiment and theory.

\section{Review of Some Experiments}

We begin by reviewing briefly some experimental findings, which leads to the simple structural picture upon which our work is based.

A. Unsonicated Lecithin Bilayers. We consider the case of unsonicated lecithin bilayers in which all the hydrocarbon chains are saturated and of a single length. Prolonged sonication produces small vesicles with altered properties 22 which do not necessarily exhibit the phase transition with which we are concerned. ${ }^{3}$ Hence, we shall focus almost exclusively on lamellar dispersions in what follows.

Below the phase transition temperatures $T_{\mathrm{m}}$ of such bilayers, $X$-ray diffraction studies show that the hydrocarbon chains are all-trans and organized in a two-dimensional hexagonal array. The $\mathrm{X}$-ray diffraction pattern indicates that the distance between neighboring chains is $4.8 \AA .{ }^{23}$ Proton NMR of these bilayers shows no high-resolution features arising from the hydrocarbon chains. ${ }^{22}$ The two chains of a given molecule may not be entirely equivalent; $\mathrm{X}$-ray diffraction studies of the related phosphatidyl ethanolamines show that one chain penetrates more deeply into the bilayer than the other. ${ }^{24}$

As the temperature is raised, calorimetric and volumetric studies show that a broad "pretransition" occurs before the main transition temperature $T_{\mathrm{m}}$ is attained. ${ }^{3,25} \mathrm{X}$-Ray dif- 
fraction studies reveal that the hydrocarbon chains change from a tilted inclination to one which is perpendicular to the plane of the bilayer, although the interchain spacing remains at $4.8 \AA,{ }^{16}$ and NMR studies show that there are changes in the mobility and hydration of the headgroups. ${ }^{6}$ It is interesting to note that phosphatidyl ethanolamine bilayers do not exhibit a "pretransition". 6

At the temperature $T_{\mathrm{m}}$, a very sharp transition is observed. 3,25 This is the transition with which we are concerned. The width of this transition is sensitive to sample purity and might vanish with completely pure lipids. ${ }^{3}$ Above $T_{\mathrm{m}}$, the $\mathrm{X}$-ray diffraction pattern shows somewhat broadened bands, suggesting scattering from clusters of hydrocarbon chains. In these clusters the chains are still fairly extended and hexagonally ordered with an average interchain spacing of about $5.3 \AA .^{23}$ Hinz and Sturtevant's analysis of the calorimetric transition widths indicates that the clusters formed upon chain melting must comprise at least 70 molecules and may be considerably larger. ${ }^{3}$ X-Ray diffraction and deuterium NMR studies both show that the hydrocarbon chain axes are oriented perpendicularly to the plane of the bilayer at temperatures above $T_{\mathrm{m} \cdot}{ }^{26,27}$ The proton NMR spectrum shows narrowed bands arising from the choline methyl groups and the terminal methyl groups of the fatty acid chains. ${ }^{22}$ The reduction of conformational restrictions on the choline groups and chain-terminal methyl groups is also apparent in the recent proton-enhanced ${ }^{13} \mathrm{C}$ NMR studies by Urbina and Waugh. ${ }^{28}$ Consideration of the proton relaxation times led Chan et al. to a model for the hydrocarbon chain motion in which the terminal methyl group and a few adjacent methylene groups are conformationally mobile (trans-gauche rotations of the neighboring bonds are relatively unrestricted). The remaining methylene groups are conformationally restricted (gauche rotations of opposite sense occur as coupled pairs or kinks which preserve the overall chain orientation and lead to relatively long correlation times of $10^{-5}$ to $\left.10^{-7} \mathrm{sec}\right){ }^{22,29,30}$ Clear evidence for the mobility of several methylenes nearest the middle of the bilayer is given in recent deuterium NMR work by Seelig and Seelig, who also noted that the two chains of a given molecule are not completely equivalent. ${ }^{31}$

B. Related Systems with Fluid Chains. The qualitative picture elaborated by Chan et al. of the hydrocarbon chain motions in fluid lecithin lamellae is supported by studies on other systems. Seelig and Niederberger studied lamellar decanol-decanoate bilayers containing decanoate molecules deuterated at specific positions along the chain. From the analysis of their deuterium NMR measurements, they found clear evidence that most of the hydrocarbon chain is conformationally restricted, the chain tending to remain perpendicular to the plane of the bilayer, but that the terminal methyl group and two neighboring methylenes experience a more nearly isotropic motion. ${ }^{32}$ In sonicated lecithin bilayers, proton and ${ }^{13} \mathrm{C} N \mathrm{~N}$ R studies reveal chain motions which are qualitatively like those of fluid lamellar bilayers, with a sudden decrease in conformational restriction at the terminal methyl group and two nearest methylenes. ${ }^{33,34}$ This behavior is also found by Belle and Bothorel in their computer simulation of chain motions, ${ }^{19}$ and is geometrically rationalized in the work of Rothman. ${ }^{18}$

Experimental evidence for the cooperative formation of kinks is given in the work of Lagaly and Weiss, who have studied $n$-alkanol complexes of $n$-alkylammonium layer silicates. $^{35}$ The alkanol and alkylammonium molecules are arranged in bimolecular layers between silica sheets, with the alkyl chains perpendicular to the silicate layers. Using $\mathrm{X}$-ray diffraction, Lagaly and Weiss found that the distance between the silicate sheets decreased in well-defined steps as the temperature was increased. The separation decre- ments were just what one would expect if the chains were forming kinks such as $\mathrm{g}^{+} \mathrm{tg}^{-}$or $\mathrm{g}^{-} \mathrm{tg}^{+}$, where $\mathrm{t}$ indicates a trans bond and $\mathrm{g}^{+}$and $\mathrm{g}^{-}$indicate gauche bonds generated by dihedral rotations of opposite sense.

C. Qualitative Picture of the Structures of the Solid and Fluid Phases of Lamellar Lecithin Bilayers. For definiteness in setting up quantitative models, we postulate the following picture of bilayer structure.

In the solid phase, the hydrocarbon chains are all-trans and hexagonally ordered with an interchain distance of 4.8 $\AA$.

In the fluid phase a "snapshot" would show the chains organized into clusters, within each of which significant order is retained. Gauche bonds only occur as paired rotations of opposite sense over most of the chain length, forming kinks which conserve the overall chain direction. This minimizes the lateral expansion of the bilayer by reducing the volume between clusters. All the chains within a given cluster have similar kink configurations, allowing a dovetailed hexagonal packing. However, several bonds nearest the terminal methyl groups can move with less steric hindrance and these terminal regions are disordered within the clusters. The two chains of a given molecule are not equivalent. One chain in each molecule penetrates more deeply into the bilayer and may experience more terminal disordering.

The solid and fluid phases are illustrated schematically in Figure 1.

Apart from the conformational freedom in the bonds nearest the ends of the hydrocarbon chains, the nature of the chain disordering in the fluid phase is assumed to be as proposed by Träuble, ${ }^{36}$ who based his ideas on the "kinkblock" model of pure hydrocarbon melts constructed by Blasenbrey and Pechold. ${ }^{37}$ Blasenbrey and Pechold's model has also been explicitly cited in rationalizing some of the NMR studies mentioned above. $22,29-33$

\section{Model for Pure, Lamellar Phospholipid Bilayer Phase Transitions}

The statistical thermodynamic formalism we adopt in this paper is equivalent to a Bragg-Williams treatment. ${ }^{44}$ To carry the program through, it is necessary to specify the internal energy states, the intermolecular interaction energies, and the coordination numbers of the phospholipid molecules. Preliminary work using reasonable estimates for the interaction energies of trans-trans, kinky-kinky, and transkinky chain pairs showed that trans and kinky chains are virtually immiscible. Trans-trans and dovetailed kinkykinky pairs are much more stable than the mismatched trans-kinky pairs, so that a model for an assembly of such chains exhibits a first-order phase transition from an alltrans state to an all-kinky state as the temperature is raised. This allows us to use a simplified approach in which separate models established for the solid, all-trans and fluid, allkinky phases. Bilayer melting occurs when the chemical potentials of molecules in the solid and fluid phases become equal.

A. Partition Function for the Solid Phase. We choose the chain conformation and head group energy scales to be zero for all-trans chains and solid-state head groups. Thus the internal partition function for molecules in the solid phase is taken to be

$$
q_{\mathrm{s}}(T)=\exp \left(-w_{\mathrm{tl}} / R T\right)
$$

where $w_{t 1}$ is the interaction energy of the two all-trans chains in the molecule.

A given phospholipid molecule in the solid phase is surrounded by eight hydrocarbon chains from its neighbors, 
leading to ten pairwise intermolecular chain interactions. ${ }^{24}$ We assume that these intermolecular chain interactions have the same strength as the intramolecular interaction $w_{\mathrm{tt}}$. Thus if we ignore the relatively rare defects in the twodimensional packing, ${ }^{39}$ the canonical partition function fornthe solid assembly of $N$ molecules is

$$
Q_{\text {solid }}(N, T)=q_{\mathrm{s}}(T)^{N} \exp \left[-\frac{N}{2 R T}\left(10 w_{\mathrm{tt}}\right)\right]
$$

or, employing eq 2.1 ,

$$
Q_{\text {solid }}(N, T)=\exp \left[-\frac{6 w_{\mathrm{tt}} N}{R T}\right]
$$

The head groups of the phospholipid molecules may be treated conceptually as asymmetrical dimers on a triangular lattice covering the hexagonal lattice of the chains. ${ }^{17}$ If these dimers are orientationally disordered, we should include an extra factor in eq 2.3 to account for the resulting configurational entropy. The X-ray studies of Hitchcock et al. ${ }^{24}$ indicate that the dimers are well ordered in the case of solid phosphatidyl ethanolamines, as shown schematically in Figure 2, and we choose not to modify eq 2.3 .

B. Partition Function for the Fluid Phase. We assume that the fluid phase is composed of a mixture of clusters. In each cluster every chain is assumed to have the same kink configuration. The internal partition function of each molecule in any given cluster must first be computed. The internal energy of the molecule $\epsilon_{l}$ in a particular cluster that contains exactly $l$ links will be considered to comprise contributions from the kinky chain conformational states, intramolecular interactions of the two dovetailed kinky chains, and alterations in the energy of the head group relative to the solid phase. Furthermore the ends of the chain are disordered and can assume all the gauche-trans conformations allowed by the "rotational isomeric state model" of Flory. ${ }^{38}$ We assume that this terminal disordering is independent of the degree of kinking in the chain. Many schemes could be proposed for the terminal disordering; however, we will consider two, yielding a less disordered and a more disordered model.

The internal partition function for a molecule in an $l$ kink cluster may then be written in the form

$$
q_{l}(T)=\exp \left(-\epsilon_{l} / R T\right) f_{\mathrm{d}}(T)
$$

where $f_{\mathrm{d}}(T)$ is the factor representing the terminal disorder. Here

$$
\epsilon_{l}=E_{\mathrm{h}}+w_{\mathrm{kk}}+2400 l
$$

where $E_{\mathrm{h}}$ is the change in head group energy relative to the solid phase, $w_{k k}$ is the intramolecular interaction energy between the two dovetailed kinked chains which is assumed to be independent of the degree of kinking, and $2400 \mathrm{l} \mathrm{cal} / \mathrm{mol}$ is the energy of $2 l\left(\mathrm{~g}^{+}, \mathrm{g}^{-}\right)$kinked pairs that arises from the $600 \mathrm{cal} / \mathrm{mol}$ cost of a single trans-to-gauche rotation. ${ }^{38}$ Thus

$q_{l}(T)=\exp \left[-\frac{1}{R T}\left(2400 l+w_{\mathrm{kk}}+E_{\mathrm{h}}\right)\right] f_{\mathrm{d}}(T)$

For the terminal disorder factor $f_{\mathrm{d}}(T)$, a model with relatively little disorder is obtained by assuming that in one chain of each molecule the two methylene-methylene bonds nearest the terminal methyl group are conformationally mobile, but that there is no mobility in the partner chain. The states of the mobile bonds which are allowed by Flory's scheme are $\mathrm{tt}, \mathrm{tg}^{+}, \mathrm{tg}^{-}, \mathrm{g}^{+} \mathrm{t}, \mathrm{g}^{-} \mathrm{t}, \mathrm{g}^{+} \mathrm{g}^{+}$, and $\mathrm{g}^{-} \mathrm{g}^{-}$. The corresponding factor is

$$
f_{\mathrm{d}}(T)=1+4 e^{-600 / R T}+2 e^{-1200 / R T}
$$

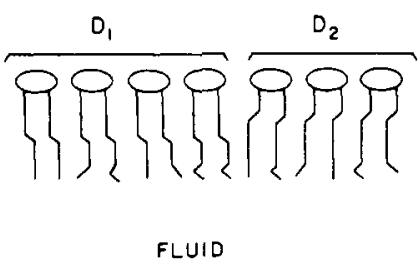

Figure 1. Schematic illustration of the hydrocarbon chain organization in half of a bilayer. In the solid state, the chains are all-trans. In the fluid state, the kinky chains are coherently organized within clusters such as $D_{1}$ and $D_{2}$. Trans-gauche disordering within the fluid state clusters occurs only in the bonds nearest the terminal methyl groups. The slight dissimilarity of the two chains in a given molecule is not shown.

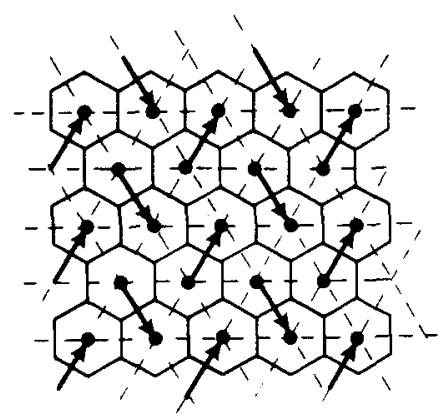

Figure 2. Schematic illustration of the head group organization in solid phosphatidyl ethanolamine. ${ }^{24}$ Head groups are represented by arrows pointing from the nitrogen atom to the phosphorus atom. The head groups can be considered as "dimers" connecting the vertices of the dotted triangular lattice. The hydrocarbon chains occupy the solid hexagons below the triangular lattice.

A model with more disordering is obtained by assuming that there are four mobile bonds in one chain and two in the other. This is closer to what Seelig and Seelig's results suggest ${ }^{31}$ and leads to the factor

$$
\begin{array}{r}
f_{\mathrm{d}}(T)=1+12 e^{-600 / R T}+52 e^{-1200 / R T}+100 e^{-1800 / R T}+ \\
86 e^{-2400 / R T}+32 e^{-3000 / R T}
\end{array}
$$

The combination of eq 2.6 and $2.7 \mathrm{a}$ or $2.7 \mathrm{~b}$ gives an expression for the internal partition function for a molecule in an $l$-kinked cluster. Next we include the interaction energy between molecules in the cluster employing the same approximations that led to eq 2.3 for the solid phase. Thus the partition function for an $l$-kinked cluster that contains $N_{l}$ molecules is simply

$$
Q_{l}\left(N_{l}, T\right)=q_{l}(T)^{N_{l}} \exp \left[-\frac{N_{l}}{2 R T}\left(10 w_{\mathrm{kk}}\right)\right]
$$

This expression may be cast in terms of an effective single molecule partition function $\bar{q}_{/}(T)$ with an associated free energy per molecule $a_{l}(T)$ :

$$
a_{l}(T)=-R T \ln \bar{q}_{l}(T)
$$

where

$$
a_{l}(T)=\epsilon_{l}+5 w_{\mathrm{kk}}-R T \ln f_{\mathrm{d}}(T)
$$

One finds

$$
Q_{l}\left(N_{l}, T\right)=\bar{q} l^{N_{l}}
$$

Our final step in constructing the partition function for the entire fluid phase requires that we take into account the various occupations $\left\{N_{l}\right\}$ that can occur in the clusters that comprise the fluid phase. There may be many clusters that contain $l$ kinks. But we neglect surface effects in the clus- 
ters, a simplification that prevents determination of this size distribution, and treat each $l$-kinked cluster class as an independent subsystem. ${ }^{44}$ Accordingly the partition function and associated free energy for the fluid phase is given by

$$
\exp \left[-A_{\mathrm{f}}(N, T) / R T\right]=Q_{\mathrm{f}}(N, T)=\sum_{\left\{N_{l}\right\}}^{\prime} N ! \prod_{l} \frac{\bar{q}_{l} N_{l}}{N_{l} !}
$$

where the prime indicates that the sum over all occupation numbers must be performed subject to the condition

$$
\sum_{l} N_{l}=N
$$

We evaluate the partition function $Q_{\mathrm{f}}(N, T)$ by the maximum term method ${ }^{44}$ and find

$$
Q_{\mathrm{f}}=N ! \prod_{l} \frac{\bar{q}_{l} N_{l}^{*}}{N_{l}^{*} !}=\exp \left[-\frac{A_{\mathrm{f}}(N, T)}{R T}\right]
$$

where the occupation numbers for the $l$-kinked clusters are given by

$$
N_{l}^{*}=N C^{-1} \exp \left(-a_{l} / R T\right)
$$

where

$$
C=\sum_{l} \exp \left(-a_{l} / R T\right)
$$

or alternatively

$$
C=\exp \left[-\frac{E_{\mathrm{h}}}{R T}-6 \frac{W_{\mathrm{kk}}}{R T}\right] f_{\mathrm{d}}(T) \sum_{l} e^{-u l}
$$

where $u=2400 / R T$. The sum runs from $l=1$ to $l_{\max }$ which we take to be infinite, incurring negligible error. Thus

$$
C=\exp \left[-\frac{E_{\mathrm{h}}}{R T}-6 \frac{w_{\mathrm{kk}}}{R T}\right] f_{\mathrm{d}}(T)\left[e^{u}-1\right]^{-1}
$$

and

$$
N_{l}^{*}=N\left(e^{u}-1\right) e^{-u l}
$$

The free energy of the fluid is evaluated for the maximum term in eq 2.12 and a simple calculation leads to

$$
Q_{\mathrm{f}}(N, T)=C^{N}=\exp \left[-A_{\mathrm{f}} / R T\right]
$$

Since we do not know the extent of dimer-type disordering in the fluid phase, we will consider the extreme cases of complete retention of order and complete randomization. In the first case eq 2.20 stands as it is, while in the second case a factor of $(4.713)^{N}$, the number of ways of ordering $N$ asymmetric dimers on a triangular lattice when edge effects are ignored, ${ }^{17,40}$ should be included on the right.

The above results can be given in terms of "effective" single molecule partition functions $q_{\text {solid }}(T)$ and $q_{\text {fluid }}(T)$ :

$$
\begin{gathered}
Q_{\text {solid }}(N, T)=q_{\text {solid }} N(T) \\
q_{\text {solid }}(T)=\exp \left(-6 w_{\mathrm{tl}} / R T\right) \\
Q_{\text {fluid }}(N, T)=q_{\text {fluid }}(T) \\
C=q_{\text {fluid }}(T)=\exp \left[-\frac{1}{R T}\left(6 w_{\mathrm{kk}}+\right.\right. \\
\left.E_{\mathrm{h}}\right) f_{\mathrm{d}}(T) f_{\mathrm{k}}(T)\left\{\begin{array}{c}
1 \\
\text { or } \\
4.713
\end{array}\right\}
\end{gathered}
$$

where we have introduced the notation

$$
f_{\mathrm{k}}(T)=\left[e^{u}-1\right]^{-1}
$$

We ignore PV terms since the molar volume changes of bilayers are very small ${ }^{25}$ and find that the chemical potentials are given by

$$
\mu=-R T\left(\frac{\partial \ln Q}{\partial N}\right)_{T}
$$

so that

$$
\mu_{\text {solid }}(T)=6 w_{\mathrm{tt}}
$$

and

$\mu_{\mathrm{fluid}}(T)=6 w_{\mathrm{kk}}+E_{\mathrm{h}}-R T \ln f_{\mathrm{d}}(T)-$

$$
R T \ln f_{\mathrm{k}}(T)-\left\{\begin{array}{c}
0 \\
\text { or } \\
R T \ln 4.713
\end{array}\right\}
$$

C. Applications. Salem, ${ }^{41}$ Zwanzig, ${ }^{42}$ and Shapiro and Ohki ${ }^{43}$ have studied the attractive force between two parallel, all-trans, saturated hydrocarbon chains. For sufficiently long chains, the force is proportional to $n$, the number of carbon atoms in the chain. The short-range repulsive force is presumed to behave similarly. ${ }^{43} \mathrm{We}$ will write the interaction energy between neighboring all-trans phospholipid chains in the form

$$
w_{\mathrm{tt}}=w_{\mathrm{tt}}^{0}(n-2)
$$

where $n-2$ is an approximate representation of the actual overlapping length of neighboring $n$-carbon chains. ${ }^{24} \mathrm{~A}$ similar expression

$$
w_{\mathrm{kk}}=w_{\mathrm{kk}}{ }^{0}(n-2)
$$

should adequately represent the interaction between dovetailed kinky chains when only a few gauche rotations are present.

Then from eq 2.24 through 2.27 ,

$\mu_{\text {fluid }}(T)-\mu_{\text {solid }}(T)=E_{\mathrm{h}}+6(n-2) \Delta w^{0}-R T \ln f_{\mathrm{d}}(T)-$

where

$$
R T \ln f_{\mathrm{k}}(T)-\left\{\begin{array}{c}
0 \\
\text { or } \\
R T \ln 4.713
\end{array}\right\}
$$

$$
\Delta w^{0}=w_{\mathrm{kk}}^{0}-w_{\mathrm{tt}}^{0}
$$

Since we have no theoretical information on the values of $E_{\mathrm{h}}$ or $w_{\mathrm{kk}}{ }^{0}$, we will use the experimental values of $T_{\mathrm{m}}$ for two bilayers with different chain lengths to determine these parameters for phospholipids with a given type of head group. This is done simply by choosing $E_{\mathrm{h}}$ and $\Delta w^{0}$ such that $\mu_{\text {fluid }}(T)-\mu_{\text {solid }}(T)=0$ for each bilayer at its $T_{\mathrm{m}}$.

1. Phosphatidyl Cholines (Lecithins). In the case of lecithins, we use the experimental $T_{\mathrm{m}}$ of the relatively wellstudied dipalmitoyl $\left(T_{\mathrm{m}}=42^{\circ} \mathrm{C}\right)$ and distearoyl $\left(T_{\mathrm{m}}=\right.$ $58^{\circ} \mathrm{C}$ ) systems for parametrization. ${ }^{3}$

For the model with less terminal disordering, where $f_{\mathrm{d}}(T)$ is given by eq $2.7 \mathrm{a}$,

$$
\Delta w^{0}=4.99 \mathrm{cal} / \mathrm{mol} \text { per carbon; } E_{\mathrm{h}}=-2.15 \mathrm{kcal} / \mathrm{mol}
$$

with the "dimer-ordered" fluid assumption, and

$$
\Delta w^{0}=9.10 \mathrm{cal} / \mathrm{mol} \text { per carbon; } E_{\mathrm{h}}=-1.53 \mathrm{kcal} / \mathrm{mol}
$$

with the "dimer-disordered" fluid assumption.

For the model with more terminal disordering, where $f_{\mathrm{d}}(T)$ is given by eq $2.7 \mathrm{~b}$,

$$
\Delta w^{0}=13.95 \mathrm{cal} / \mathrm{mol} \text { per carbon; } E_{\mathrm{h}}=-1.65 \mathrm{kcal} / \mathrm{mol}
$$

$(2.30 \mathrm{c})$ 
with the "dimer-ordered" fluid assumption, and

$$
\Delta w^{0}=18.06 \mathrm{cal} / \mathrm{mol} \text { per carbon; } E_{\mathrm{h}}=-1.03 \mathrm{kcal} / \mathrm{mol}
$$

with the "dimer-disordered" fluid assumption.

The predicted melting temperatures for the series of lecithin bilayers are compared with the experimental results in Figure 3. The theoretical curve is based on the parameters of eq $2.30 \mathrm{c}$, but the other parameter sets yield virtually the same results.

The energy of the fluid and solid phases at the transition temperature may be calculated from the canonical partition functions by standard methods ${ }^{44}$ From these, the energy and entropy of transition may be calculated. For dipalmitoyl lecithin and the more terminal-disordered model, the results are

$$
\Delta E=3.27 \mathrm{kcal} / \mathrm{mol}, \Delta S=10.4 \mathrm{eu}
$$

with the "dimer-ordered" assumption, and

$$
\Delta E=4.24 \mathrm{kcal} / \mathrm{mol}, \Delta S=13.5 \mathrm{eu}
$$

with the "dimer-disordered" assumption. These results are only about 30 to $40 \%$ of the experimental values, ${ }^{3}$ but should be significantly improved by extending the idealized models treated here to include the additional fluid energy and disorder arising from the boundary effects of the clusters and head group mobility.

2. Phosphatidyl Ethanolamines. The melting characteristics of phospholipids other than the lecithins are not well established. Reported transition temperatures for the phosphatidyl ethanolamines cover a wide range, reflecting different experimental conditions. Ladbrooke and Chapman studied the series $C_{14}, C_{16}$, and $C_{18}$ and found melting temperatures of approximately 77,83 , and $88^{\circ} \mathrm{C}$ for the aqueous dispersions. ${ }^{45}$ For this system, the melting parameters are

$$
\Delta w^{0}=4.47 \mathrm{cal} / \mathrm{mol} \text { per carbon, } E_{\mathrm{h}}=-0.42 \mathrm{kcal} / \mathrm{mol}
$$

where we have used the more terminal disordered model and assumed no "dimer disordering" in the fluid. We will comment below on the difference between these numbers and those in eq $2.30 \mathrm{c}$.

\section{Model for Two-Component Phospholipid Bilayer Phase Separations}

A major motivation of this work has been our interest in studying mixed bilayers, which bear a closer resemblance to biological membranes than do pure bilayers. In this section we show how models for two-component bilayers can be developed along the lines established in section III.

As in the preceding section, we will treat the solid and fluid phases separately. We will derive expressions for the chemical potentials of the two components, $A$ and $B$, in each phase and show how a phase diagram may be constructed by finding phase compositions which satisfy equilibrium requirements at each temperature.

We avoid a lengthy, though straightforward, derivation by starting with the usual form of the Bragg-Williams equations for chemical potentials in two-component systems. ${ }^{44}$ Equations appropriate for the present purpose are then obtained by a reinterpretation of certain parameters. The usual Bragg-Williams expression for component $\mathbf{A}$ is

$$
\begin{array}{r}
\mu_{\mathrm{A}}\left(X_{\mathrm{A}}, T\right)=R T \ln X_{\mathrm{A}}-R T \ln q_{\mathrm{A}}+\frac{c}{2} \epsilon_{\mathrm{AA}} X_{\mathrm{A}}+ \\
\frac{c}{2} X_{\mathrm{B}}\left(X_{\mathrm{A}} \epsilon_{\mathrm{AA}}-X_{\mathrm{B}} \epsilon_{\mathrm{BB}}+2 X_{\mathrm{B}} \epsilon_{\mathrm{AB}}\right)
\end{array}
$$

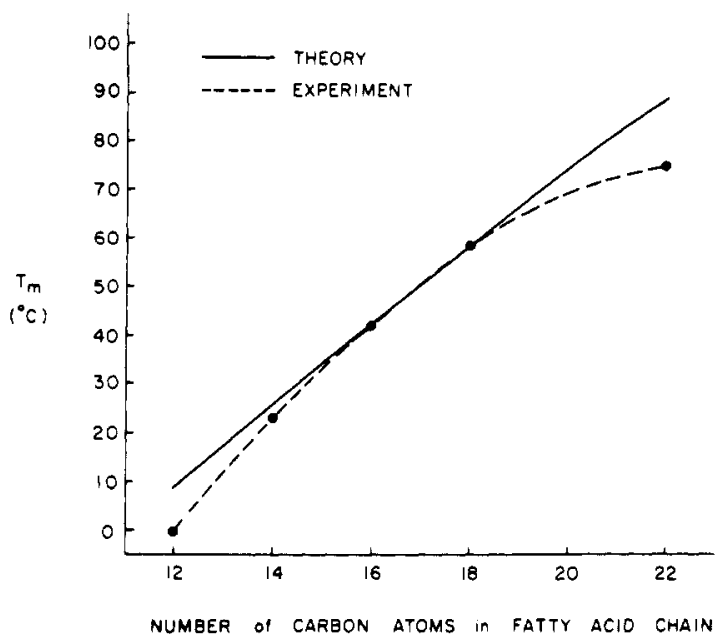

Figure 3. Experimental melting temperatures of saturated chain lecithin bilayers $2 a .3 .4$ compared with melting temperatures predicted by the theory. The experimental temperatures obtained by various workers for a given lipid cover a range of up to $3^{\circ} \mathrm{C}$, reflecting differences in sample hydration.

where $X_{\mathrm{A}}$ and $X_{\mathrm{B}}=1-X_{\mathrm{A}}$ are the mole fractions of components $A$ and $B ; \epsilon_{A A}, \epsilon_{B B}$, and $\epsilon_{A B}$ are interaction energies between pairs of molecules; $c$ is the number of nearest neighbors around a given molecule, and $q_{\mathrm{A}}$ is the internal partition function of molecule $A$. The equation for $\mu_{\mathrm{B}}\left(X_{\mathrm{A}}, T\right)$ follows from eq 3.1 by interchanging $\mathrm{A}$ and $\mathrm{B}$ subscripts on the right-hand side. Now, because each phospholipid molecule has two chains in the hexagonal lattice, a given molecule can have from four to eight neighbors. On the average, however, each molecule will be in contact with six others and will interact with both chains of two of these neighbors and with one chain of each of the remaining four (see Figure 2). A typical molecule will thus have ten chainpair interactions with six neighboring phospholipids, and we take

$c=6, \epsilon_{\mathrm{AA}}=\frac{10}{6} w^{\mathrm{AA}}, \epsilon_{\mathrm{BB}}=\frac{10}{6} w^{\mathrm{BB}}, \epsilon_{\mathrm{AB}}=\frac{10}{6} w^{\mathrm{AB}}$

where $w^{\mathrm{AA}}, w^{\mathrm{BB}}$, and $w^{\mathrm{AB}}$ are respectively the interaction energies between two $A$ molecule chains, between two $B$ molecule chains, and between an A molecule chain and a B molecule chain. Substituting eq 3.2 into eq 3.1 yields

$$
\begin{array}{r}
\mu_{\mathrm{A}}\left(X_{\mathrm{A}}, T\right)=R T \ln X_{\mathrm{A}}-R T \ln q_{\mathrm{A}}+5 w^{\mathrm{AA}} X_{\mathrm{A}}+ \\
5 X_{\mathrm{B}}\left(X_{\mathrm{A}} w^{\mathrm{AA}}-X_{\mathrm{B}} w^{\mathrm{BB}}+2 X_{\mathrm{B}} w^{\mathrm{AB}}\right)
\end{array}
$$

and an equation for $\mu_{\mathrm{B}}\left(X_{\mathrm{A}}, T\right)$ by interchange of $\mathrm{A}, \mathrm{B}$ on the right-hand side. Here we employ the effective internal partition functions

$$
\begin{aligned}
& q_{\mathrm{A}}=e^{-w_{\mathrm{t}}^{\mathrm{AA}} / R \mathrm{~T}} \\
& q_{\mathrm{B}}=e^{-w_{\mathrm{t}}^{\mathrm{BB}} / R T}
\end{aligned}
$$

for the solid phase, and

$$
\begin{aligned}
q_{\mathrm{A}} & =e^{-w_{\mathrm{k}} A \cdot A / R T} e^{-E_{\mathrm{h}} \mathrm{A} / R T} f_{\mathrm{d}} \mathrm{A} f_{\mathrm{k}} \\
q_{\mathrm{B}} & =e^{-w_{\mathrm{k}} \mathrm{BB} / R T} e^{-E_{\mathrm{h}} \mathrm{B} / R T} f_{\mathrm{d}}{ }^{\mathrm{B}} f_{\mathrm{k}}
\end{aligned}
$$

for the fluid phase. $w_{t}{ }^{A A}, w_{t}{ }^{B B}$, and $w_{t}{ }^{A B}$ are interaction energies between neighboring all-trans chains; $w_{k}{ }^{A A}, w_{k}{ }^{B B}$, and $w_{k}{ }^{A B}$ are interaction energies between dovetailed kinky chains; $f_{\mathrm{d}}{ }^{A}(T)$ and $f_{\mathrm{d}}{ }^{\mathrm{B}}(T)$ are the "terminal disordering" functions as in eq 2.7 , where we have allowed for differences in the $\mathrm{A}$ and $\mathrm{B}$ chains; $f_{\mathrm{k}}(T)$ is the kinking function given by eq $2.22 \mathrm{c}$; and $E_{\mathrm{h}}{ }^{\mathrm{A}}, E_{\mathrm{h}}{ }^{\mathrm{B}}$ are the head group energy 


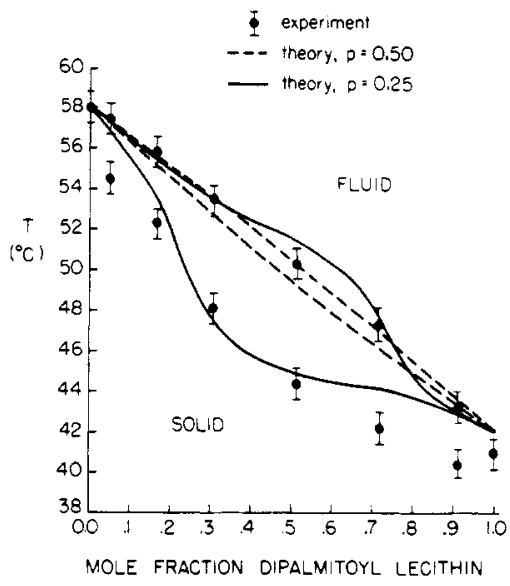

Figure 4. Theoretical phase diagrams for dipalmitoyl lecithin and distearoyl lecithin bilayers, compared with experimental points. ${ }^{4} p$ is the parameter appearing in eq 3.13a. Experiments performed under different conditions show the same general phenomenon, but yield slightly different curves. ${ }^{5}$

parameters for phospholipids A and B. For the fluid phase these effective internal partition functions differ from the single molecule fluid partition function, eq $2.22 \mathrm{~b}$, by elimination of the intermolecular interaction energy $5 w_{\mathrm{kk}}$. Thus we finally obtain

$$
\begin{aligned}
& \mu_{\mathrm{A}}{ }^{\mathrm{s}}\left(X_{\mathrm{A}}^{\mathrm{s}}, T\right)=R T \ln X_{\mathrm{A}}^{\mathrm{s}}+\left(1+5 X_{\mathrm{A}}^{\mathrm{s}}\right) w_{\mathrm{t}}{ }^{\mathrm{AA}}+ \\
& 5 X_{\mathrm{B}}^{\mathrm{s}}\left(X_{\mathrm{A}}{ }^{\mathrm{s}} w_{\mathrm{t}}{ }^{\mathrm{AA}}-X_{\mathrm{B}}{ }^{\mathrm{s}} w_{\mathrm{t}}^{\mathrm{BB}}+2 X_{\mathrm{B}}{ }^{\mathrm{s}} w_{\mathrm{t}}{ }^{\mathrm{AB}}\right) \\
& \mu_{\mathrm{B}}{ }^{\mathrm{s}}\left(X_{\mathrm{A}}^{\mathrm{s}}, T\right)=R T \ln X_{\mathrm{B}}{ }^{\mathrm{s}}+\left(1+5 X_{\mathrm{B}}^{\mathrm{s}}\right) w_{\mathrm{t}}{ }^{\mathrm{BB}}+ \\
& 5 X_{\mathrm{A}}{ }^{\mathrm{s}}\left(X_{\mathrm{B}}{ }^{\mathrm{s}} w_{\mathrm{t}}{ }^{\mathrm{BB}}-X_{\mathrm{A}}{ }^{\mathrm{s}} w_{\mathrm{t}}^{\mathrm{AA}}+2 X_{\mathrm{A}}{ }^{\mathrm{s}} w_{\mathrm{t}}{ }^{\mathrm{AB}}\right)
\end{aligned}
$$

for the chemical potentials in the solid phase, and

$$
\begin{array}{r}
\mu_{\mathrm{A}}^{\mathrm{f}}\left(X_{\mathrm{A}}^{\mathrm{f}}, T\right)=R T \ln X_{\mathrm{A}}^{\mathrm{f}}+\left(1+5 X_{\mathrm{A}}{ }^{\mathrm{f}}\right) w_{\mathrm{k}}{ }^{\mathrm{AA}}+ \\
5 X_{\mathrm{B}}{ }^{\mathrm{f}}\left(X_{\mathrm{A}}{ }^{\mathrm{f}} w_{\mathrm{k}}{ }^{\mathrm{AA}}-X_{\mathrm{B}}{ }^{\mathrm{f}} w_{\mathrm{k}}{ }^{\mathrm{BB}}+2 X_{\mathrm{B}}{ }^{\mathrm{f}} w_{\mathrm{k}}{ }^{\mathrm{AB}}\right)- \\
R T \ln f_{\mathrm{d}}^{\mathrm{A}}(T)-R T \ln f_{\mathrm{k}}(T)+E_{\mathrm{h}}^{\mathrm{A}} \\
\mu_{\mathrm{B}}{ }^{\mathrm{f}}\left(X_{\mathrm{A}}{ }^{\mathrm{f}}, T\right)=R T \ln X_{\mathrm{B}}^{\mathrm{f}}+\left(1+5 X_{\mathrm{B}}{ }^{\mathrm{f}}\right) w_{\mathrm{k}}{ }^{\mathrm{BB}}+ \\
5 X_{\mathrm{A}}^{\mathrm{f}}\left(X_{\mathrm{B}}{ }^{\mathrm{f}} w_{\mathrm{k}}{ }^{\mathrm{BB}}-X_{\mathrm{A}}{ }^{\mathrm{f}} w_{\mathrm{k}}{ }^{\mathrm{AA}}+2 X_{\mathrm{A}}{ }^{\mathrm{f}} w_{\mathrm{k}}{ }^{\mathrm{AB}}\right)- \\
R T \ln f_{\mathrm{d}}^{\mathrm{B}}(T)-R T \ln f_{\mathrm{k}}(T)+E_{\mathrm{h}}^{\mathrm{B}}
\end{array}
$$

for the chemical potentials in the fluid phase. Here $X_{\mathrm{A}}^{\mathrm{s}}$ and $X_{A}{ }^{f}$ are the mole fractions of $A$ in the solid and fluid phases, respectively, $X_{\mathrm{B}}{ }^{\mathrm{s}}=1-X_{\mathrm{A}}{ }^{\mathrm{s}}$ and $X_{\mathrm{B}}{ }^{\mathrm{f}}=1-X_{\mathrm{A}}{ }^{\mathrm{f}}$ are the corresponding mole fractions of $B$. One could also include a "dimer-disordering" term in eq 3.7.

The conditions for thermodynamic stability of the A,B system at temperature $T$ are

$$
\begin{aligned}
& \mu_{\mathrm{A}}^{\mathrm{s}}\left(X_{\mathrm{A}}^{\mathrm{s}}, T\right)=\mu_{\mathrm{A}}{ }^{\mathrm{f}}\left(X_{\mathrm{A}}^{\mathrm{f}}, T\right) \\
& \mu_{\mathrm{B}}^{\mathrm{s}}\left(X_{\mathrm{A}}^{\mathrm{s}}, T\right)=\mu_{\mathrm{B}}{ }^{\mathrm{f}}\left(X_{\mathrm{A}}^{\mathrm{f}}, T\right)
\end{aligned}
$$

These two equations in the two unknowns $X_{\mathrm{A}}^{\mathrm{s}}, X_{\mathrm{A}}^{\mathrm{f}}$ may be solved graphically for various temperatures over the range of interest to construct the phase diagram of the A,B system.

As a preliminary investigation of this approach to mixed bilayers, we have considered a system in which the two components have a common head group but different chain lengths. The particular system we chose was the dipalmitoyl lecithin-distearoyl lecithin system, for which experimental data are available, 4,5

For the interaction energy between chains of the same length, we use eq 2.26 and 2.27. A numerical expression for the attractive potential between two all-trans chains has been given by Salem ${ }^{41}$ as

$$
w(r)=-1240 n r^{-5} \mathrm{kcal} / \mathrm{mol}
$$

where $n$ is the number of carbon atoms in each chain and $r$ is the distance between the chains in angströms. For simplicity, we assume that the repulsive potential is proportional to $r^{-10}$ (Shapiro and Ohki suggest $r^{-1143}$ ). Then the interchain potential may be written in the form

$$
u_{\mathrm{tt}}(r)=\epsilon\left[\left(\frac{r^{*}}{r}\right)^{10}-2\left(\frac{r^{*}}{r}\right)^{5}\right]
$$

Using Salem's formula and $r^{*}=4.8 \AA$, it follows that $\epsilon=$ $243.3 n \mathrm{cal} / \mathrm{mol}$, and the coefficient in eq 2.26 is

$$
w_{\mathrm{tt}}{ }^{0}=-243.3 \mathrm{cal} / \mathrm{mol} \text { per carbon }
$$

If we use the lecithin parameter $\Delta w^{0}$ given in eq $2.30 \mathrm{c}$ with eq 2.29, we find that the coefficient in eq 2.27 is

$$
w_{\mathrm{kk}}{ }^{0}=-229.4 \mathrm{cal} / \mathrm{mol} \text { per carbon }
$$

New questions arise in considering the interaction energy between chains of different length. Theories of all-trans alkane chain interactions suggest that the attractive potential between two chains of slightly different length should, in vacuo, be approximately equal to the average of the interaction between two of the longer chains and the interaction between two of the shorter chains. That is, if B chains are longer than A chains,

$$
w^{\mathrm{AB}}=w^{\mathrm{AA}}+p \Delta
$$

where

$$
\Delta=w^{\mathrm{BB}}-w^{\mathrm{AA}}
$$

with $p=0.5$. The effective dielectric constant of neighboring chains might, however, be expected to diminish the factor $p$ somewhat, so we have explored the range of values $p=0.0$ to $p=0.5$.

Although some difference in the $A$ and $B$ chain terminal disordering is to be expected, we assumed $f_{\mathrm{d}} \mathrm{A}(T)=f_{\mathrm{d}}^{\mathrm{B}}(T)$ and used eq $2.7 \mathrm{~b}$ for these functions. Finally, we used the head group parameter given in eq $2.30 \mathrm{c}$ for $E_{\mathrm{h}}{ }^{\mathrm{A}}$ and $E_{\mathrm{h}}{ }^{\mathrm{B}}$.

The results of our calculations for the dipalmitoyl lecithin-distearoyl lecithin system are given in Figure $4 .{ }^{49}$ Using $p=0.5$ in eq $3.13 \mathrm{a}$, which is equivalent to assuming ideal mixing in this Bragg-Williams model ${ }^{44}$ of dipalmitoyl and distearoyl lecithins within each phase, leads to a narrow region of two-phase coexistence. Smaller values of $p$ lead to wider regions of coexistence and greater curvature of the "solidus" and "liquidus" lines, reflecting the nonideality of the solid and fluid mixtures. Values of $p$ below about 0.2 lead to immiscibility within the solid and fluid phases.

\section{Discussion}

Models which explicitly recognize changes in head group interactions in bilayer phase transitions will allow for analysis of some of the most interesting properties of biological membranes. The work described here represents a step in this direction. In this connection, it is interesting to compare the parameters derived for phosphatidyl choline and phosphatidyl ethanolamine bilayers. X-Ray and NMR studies of these systems indicate that the choline head groups are oriented perpendicularly to the plane of the bilayer, while the smaller ethanolamine head groups lie in a plane parallel to bilayer. ${ }^{46}$ These same studies suggest that the choline head group dipoles repel each other more strongly than do the ethanolamine head group dipoles, and also that there is a greater increase in hydration of the choline head groups than of the ethanolamine head groups during bilayer melting. To the extent that the $E_{h}$ parameter adequately represents changes in the free energy of the head group system, our results are consistent with this picture. The choline $E_{\mathrm{h}}$ is more negative than the ethanolam- 
ine $E_{\mathrm{h}}$, indicating greater head group stabilization for the transition to the fluid state for the cholines. In addition, the parameter $\Delta w^{0}=w_{k k}{ }^{0}-w_{t t}{ }^{0}$ is more positive for cholines than for ethanolamines, which would be consistent with the greater repulsion between choline head groups causing a slightly greater average chain separation upon melting. Important effects arising from such factors as finite cluster size and head group conformational mobility have not yet been included in these models, however, so the physical significance of the parameters $E_{\mathrm{h}}$ and $\Delta w^{0}$ cannot be as simple as we have assumed above.

Future development of models of the kind introduced in this paper should perhaps begin with inclusion of effects associated with finite cluster size in the fluid state. At least two general approaches are conceivable. One could rely on experimental estimates of an average cluster size and estimates of the attractive energy across cluster boundaries to continue the ad hoc construction of partition functions initiated here. Alternatively, estimates of cluster size and surface energy distributions (together with the frequency and energy of point defects within clusters) might be drawn from more detailed models of chain systems as such models are developed. There is also much room for improving our treatment of the head group system, which could be resolved into intramolecular (conformational mobility) and intermolecular (dipolar interaction, hydration) factors as we have done here with the chain system. The conformations of chain-terminal regions and their interactions with the opposite side of the bilayer in mixed chain-length systems also needs to be examined more closely.

With future work in experiment and theory, it should be possible to consider mixtures of phospholipids with different head groups. Data from experiments done under conditions of constant water concentration with lipids of high purity would be necessary to determine the head group interaction parameters. Alternatively, theories considering the dipolar and hydrogen bonding interactions of the head groups might yield estimates of these parameters. The isothermal modulation of membrane fluidity by hydrogen ions and divalent cations, which are known to interact selectively with certain head groups, ${ }^{9,47}$ might also be treated along these lines.

As more thermodynamic experimental data become available, the physical chemist studying lipid bilayers will face an increasingly serious dilemma. He must seek a microscopic model that reproduces on a quantitative basis measurements that reflect the highly subtle and complex organization of the membrane. A microscopic model that does justice to the complexity of the structure will quickly become intractable and its use is perhaps not justified by the accuracy of the data. Alternatively, a highly oversimplified model such as the semiempirical ones explored above may provide partial insight and allow practical analysis of data within a simple conceptual scheme. Simple models, however, cannot be expected to achieve quantitative accuracy or provide entirely satisfying answers to detailed questions concerning structure and mechanism. There is an urgent need for both types of models in order to discipline our speculation about the molecular structure of these important membrane systems.

Note Added in Proof. Recently two articles ${ }^{50,51}$ relevant to this inquiry have been drawn to our attention. The model of Andersen ${ }^{51}$ et al. is particularily noteworthy. While more sophisticated than the approach presented here, it appears to be based on a similar physical picture.
Acknowledgment. A National Science Foundation predoctoral fellowship to J. A. McCammon is gratefully acknowledged. We have enjoyed useful discussions with Professors John Waugh and George Whitesides, and Professor Martin Karplus has stimulated our collaboration. One of us, J. M. Deutch, thanks the Chemistry Department at Harvard for its hospitality during the academic year 1974-1975. The National Science Foundation provided partial support for this research.

\section{References and Notes}

(1) John Siman Guggenheim Memorial Fellow 1974-1975

(2) (a) D. Chapman, R. M. Williams, and B. D. Ladbrooke, Chem. Phys. Lipids, 1, 445 (1967); (b) D. Chapman, "Biological Membranes", Vol. 1, D. Chapman, Ed., Academic Press, New York, N.Y., 1968

(3) H.-J. Hinz and J. M. Sturtevant, J. Biol. Chem., 247, 6071 (1972).

(4) M. C. Phillips, B. D. Ladbrooke, and D. Chapman, Biochim. Biophys. Acta, 196, $35(1970)$.

(5) E. J. Shimshick and H. M. McConnell, Biochemistry, 12, 2351 (1973).

(6) D. Chapman, J. Urbina, and K. M. Keough, J. Biol. Chem., 249, 2512 (1974).

(7) R. N. McElhaney, J. Mol. Biol., 84, 145 (1974).

(8) P. Cuatrecasas, Annu. Rev. Biochem. 43, 169 (1974)

(9) H. Träuble and H. Eibl, Proc. Natl. Acad. Sci. U.S., 71, $214(1974)$

(10) S. J. Singer, Annu. Rev. Blochem., 43, 805 (1974).

(11) D. Njus. F. M. Sulzman, and J. W. Hastings, Nature (London), 248, 116 (1974).

(12) R. L. Jackson and A. M. Gotto, Jr., N. Engl. J. Med., 290, 1 (1974)

(13) R. L. Jackson and A. M. Gotto, Jr., N. Engl. J. Med., 290, 87 (1974).

(14) J. R. Trudell, W. L. Hubbell, and E. N. Cohen, Ann. N.Y. Acad. Sci, 222, 530 (1973).

(15) D. M. Small and G. G. Shipley, Science, 185, 222 (1974).

(16) B. Cater, D. Chapman, S. Hawkes, and J. Saville, Biochim. Biophys. Acta, 363, $54(1974)$

(17) J. F. Nagle, J. Chem. Phys., 58, 252 (1973).

(18) J. E. Rothman, J. Theor. Biol., 38, 1 (1973).

(19) J. Belle and P. Bothorel, Biochem. Biophys. Res. Commun., 58, 433 (1974).

(20) H. L. Scott, Jr., J. Theor. Biol., 46, 241 (1974); J. Chem. Phys., 62, 1347 (1975)

(21) S. Marcelja, Biochim. Biophys. Acta, 367, 165 (1974).

(22) S. I. Chan, M. P. Sheetz, C. H. A. Seiter, G. W. Feigenson, M. Hsu, A. Lau, and A. Yau, Ann. N.Y. Acad. Sci., 222, 499 (1973).

(23) Y. K. Levine, "X-Ray Diffraction Studies of Membranes," Pergamon Press, Elmsford, N.Y., 1973.

(24) P. Hitchcock, R. Mason, K. Thomas, and G. Shipley, Proc. Natl. Acad. Sci. U.S., 71, 3036 (1974).

(25) J. F. Nagle, Proc. Natl. Acad. Sci. U.S., 70, 3443 (1973).

(26) Y. K. Levine and M. H. F. Wilkins, Nature (London), New Biol., 230, 69 (1971).

(27) J. Seelig and A. Seelig, Biochem. Biophys. Res. Commun., 57, 406 (1974)

(28) J. Urbina and J. Waugh, Proc. Natl. Acad. Sci. U.S., 71, 5062 (1974)

(29) C. H. A. Seiter and S. I. Chan, J. Am. Chem. Soc., 95, 7541 (1973).

(30) G. W. Feigenson and S. I. Chan, J. Am. Chem. Soc., 96, $1312(1974)$

(31) A. Seelig and J. Seelig, Biochemistry, 13, 4839 (1974).

(32) J. Seelig and W. Niederberger, Biochemistry, 13, 1585 (1974).

(33) A. F. Horowitz, M. P. Klein, D. M. Michaelson, and S. J. Kohler, Ann. N.Y. Acad. Sci, 222, 468 (1973).

(34) A. G. Lee, N. J. M. Birdsall, and J. C. Metcalfe, Chem. Br., 9, 116 (1973).

(35) G. Lagaly and A. Weiss, Angew. Chem., Int. Ed. Engl., 10, 558 (1971)

(36) H. Träuble, Biomembranes, 3, 197 (1972).

(37) S. Blasenbrey and W. Pechold, Ber. Bunsenges. Phys. Chem., 74, 784 (1970).

(38) P. J. Flory, "Statistical Mechanics of Chain Molecules', Wiley, New York, N.Y., 1969.

(39) S. Hui, D. Parsons, and M. Cowden, Proc. Natl. Acad. Sci. U.S., 71 , $5068(1974)$

(40) J. Nagle, J. Chem. Phys., 152, $190(1966)$.

(41) L. Salem, J. Chem. Phys., 37, 2100 (1962).

(42) R. Zwanzig, J. Chem. Phys., 39, 2251 (1963)

(43) E. Shapiro and S. Ohki, J. Colloid interface Sci., 47, 38 (1974).

(44) T. L. Hill, "An Introduction to Statistical Thermodynamics", AddisonWesley, Reading, Mass., 1960

(45) B. D. Ladbrooke and D. Chapman, Chem. Phys. Lipids, 3, 304 (1969)

(46) M. C. Phillips, E. G. Finer, and H. Hauser, Biochim. Biophys. Acta, 290, 397 (1972)

(47) A. Verkleij, B. DeKruyff, P. Ververgaert, J. Tocanne, and L. Van Deenen, Biochim. Biophys. Acta, 339, 432 (1974).

(48) The terms "fluid" and "solid" which are not entirely appropriate for these liquid crystalline phases are used (without quotation marks) for convenience.

(49) To ensure consistency with the results of section III. it was necessary to use parameters which were more accurate than the rounded-off values reported in the text.

(50) D. Marsh, J. Membr. Biol., 18, 145 (1974)

(51) R. E. Jacobs, B. Hudson, and H. C. Andersen, Proc. Nat. Acad Sci. U.S., to be published. 